\title{
Participación chilena en reuniones IADR mundiales, 2001-2012
}

\section{Chilean participation in IADR general sessions, 2001-2012}

\author{
Cartes-Velásquez $\mathrm{R}^{1,2}$
}

\begin{abstract}
RESUMEN
Introducción: Las reuniones mundiales de la International Association for Dental Research (IADR) representan una de las instancias más importantes para la divulgación de las investigaciones odontológicas a nivel de congresos. El objetivo del presente trabajo es caracterizar la participación de investigadores de instituciones chilenas en reuniones IADR mundiales durante el período 2001-2012. Metodología: Estudio bibliométrico, la búsqueda se realizó en la plataforma web Conference Exchange ${ }^{\mathrm{TM}}$ para IADR, para las reuniones mundiales de 2001 a 2012 y el término libre "Chile", incluyendo todas las presentaciones con al menos un autor afiliado a una institución chilena; se consideraron las variables: Reunión, autor, institución, cooperación internacional y área temática. Resultados: Se hallaron 158 presentaciones, promedio de 13.2 (15.3) anuales, con participación de 1 a 6 autores en el 87.3\%; la mayor participación corresponde a la Universidad de Chile (43\%), seguida por las Universidades de Talca (15.8\%), Finis Terrae (11.4\%) y de los Andes (10.8\%); según área temática destaca Periodoncia (13.9\%), Cariología (12.7\%) y Epidemiología (12.7\%); $26 \%$ de las presentaciones tenía participación de instituciones extranjeras. Discusión: Los presentes resultados van en línea con reportes bibliométricos recientes en el área, aunque destaca la aparición de 2 universidades privadas en los primeros lugares. La valoración de la participación en las reuniones IADR mundiales representa un indicador valioso de la producción científica de la odontología chilena, que complementa las evaluaciones bibliométricas de publicaciones ISI, permitiendo una mejor comprensión de la generación de conocimiento en el área.
\end{abstract}

Rev. Clin. Periodoncia Implantol. Rehabil. Oral Vol. 6(1); 29-32, 2013.

Palabras clave: IADR, congreso, producción científica, Chile, bibliometría.

\section{ABSTRACT}

Introduction: International Association for Dental Research (IADR) general session is one of the most important annual congress in the dental and craniofacial field. The aim of this research is to characterize the participation of dental researchers affiliated to Chilean institutions in IADR general sessions over 2001-2012 period. Methodology: Bibliometric study. Search was performed in the web platform Conference Exchange ${ }^{\mathrm{TM}}$ for IADR, for general sessions from 2001 to 2012, for the term "Chile", including all presentations with at least one author with an affiliation to some Chilean institutions. The following variables were evaluated: Session, author, institution, international cooperation and topic area. Results: 158 presentations were found, annual average of $13.2(15.3)$, in $87.3 \%$ there were 1 to 6 authors per presentation. Major participant institution was Universidad de Chile (43\%), followed by Universities of Talca (15.8\%), Finis Terrae (11.4\%) and los Andes (10.8\%); topics with more participations were Periodontology (13.9\%), Cariology (12.7\%) and Epidemiology (12.7\%); $26 \%$ of the presentations had participation of foreign institutions. Discussion: These results reinforce previous bibliometrics reports, but highlight two private universities in the top positions. The assessment of participation in IADR General Sessions is an important index of scientific production of Chilean dentistry, complementing the bibliometric assessments focused on ISI publications, allowing a better understanding of knowledge generation in this field.

Rev. Clin. Periodoncia Implantol. Rehabil. Oral Vol. 6(1); 29-32, 2013.

Key words: IADR, congress, scientific production, Chile, bibliometry.

\section{INTRODUCCIÓN}

La divulgación de los resultados que se obtienen de una investigación que utiliza el método científico, es parte integral del "hacer ciencia" y no un mero proceso accesorio al trabajo de campo, laboratorio $u$ otro(1). En este sentido, las presentaciones en congresos ${ }^{(2)}$ y las publicaciones en revistas científicas ${ }^{(3)}$ aparecen como las vías de divulgación más utilizadas, siendo, en muchas ocasiones, las primeras un paso previo a las segundas ${ }^{(4)}$, mostrando tasas, tiempos y formas de publicación, desde la presentación, variables ${ }^{(5)}$.

Diversas investigaciones bibliométricas en odontología han puesto el foco en la relación que existe entre estas 2 formas de divulgación ${ }^{(6,7)}$, resaltando la importancia que tienen las presentaciones en congresos no solo como trampolín hacia la publicación en revistas, si no como un indicador valioso para estimar la productividad científica que ha desarrollado una sociedad científica ${ }^{(8)}$, país(6) o disciplina ${ }^{(7)}$.

En odontología contamos con una amplia variedad de congresos científicos, jornadas de investigación y similares, tanto a nivel de especialidades, generales, nacionales e internacionales, que se desarrollan año a año, ofreciendo la posibilidad de presentar los resultados del quehacer científico odontológico y estrechar lazos con el resto de la comunidad de investigadores, lo que favorece la realización de estudios multicéntricos y la co-autoría internacional, situación que se ha relacionado la generación de investigaciones de mayor impacto ${ }^{(9)}$.

Las reuniones de la IADR (Asociación Internacional para la Investigación Dental, International Association for Dental Research), tanto de sus secciones, divisiones, regiones, pero especialmente sus sesiones generales (mundiales), representan una de las instancias líderes para la divulgación y generación de redes de investigación en el ámbito odontológico(5).

Recientes investigaciones, de la producción científica ISI de la odontología chilena en la última década, han mostrado un aumento de esta a lo largo del período, con una fuerte concentración en su autoría y áreas temáticas ${ }^{(10)}$, así como el escaso impacto que ha tenido el aumento de escuelas de odontología en el país ${ }^{(11)}$, esto corresponde a una parte importante del panorama, sin embargo, no da cuenta de otras formas de divulgación, como son las presentaciones en congresos.

El objetivo del presente trabajo es caracterizar la participación

1. Centro de $I+D$ en Odontología Social. CIDOS.org.

2. Facultad de Odontología, Universidad de Concepción. Chile.

Correspondencia autor: Ricardo Cartes-Velásquez. ricardo@cartesvelasquez.com. Trabajo recibido el 25/10/2012. Aprobado para su publicación el 01/03/2013. 
de investigadores de instituciones chilenas en las sesiones generales de IADR durante el período 2001-2012.

\section{MATERIALES Y MÉTODOS}

La presente, corresponde a una investigación con diseño bibliométrico de tipo descriptivo. Para lo anterior se realizó una búsqueda en la plataforma Conference Exchange ${ }^{\mathrm{TM}}$ para IADR, disponible en http:// iadr.confex.com/iadr/htsearch.cgi?, en base al término libre "Chile" y restringida a las sesiones generales del período 2001-2012, esto permite la comparación con investigaciones recientes en esta temática ${ }^{(10,11)}$.

Se incluyeron todas las presentaciones con al menos un autor que indicara su afiliación a una institución chilena. Se excluyeron todos aquellos resultados repetidos e investigaciones que solo incluían a población chilena como objeto de estudio o referencia, pero sin participación de investigadores de instituciones chilenas.

Las variables incluidas corresponden a:

Sesión: Lugar y año en que la reunión fue realizada.

Título: Nombre de la presentación.

Autores: Incluyendo el primer apellido y las iniciales, generalmente solo se indicaba una sola.

Institución: Centros nacionales mencionadas en el campo de afiliación de los autores.

Cooperación Internacional: Países de los investigadores extranjeros en co-autoría.

Área: Campo o especialidad de investigación odontológica mayor, a la que suscribía la presentación en su resumen.

La tabulación de los datos se realizó en el software MS Excel 2003. Para el análisis exploratorio se utilizó el paquete estadístico STATA 10/SE con el que se generó estadística descriptiva. Los resultados se presentan mediante tablas de frecuencia y porcentajes, así como promedios con desviaciones estándar y mediana con rango intercuartílico (RIC).

\section{RESULTADOS}

Para el período estudiado se encontraron un total de 432 resúmenes, de los cuales se eliminaron 262 por repetición y 12 por no incluir autores de instituciones chilenas; con lo que el número total de presentaciones para el período 2001-2012 fue de 158, con un promedio de 13.2 (15.3) por cada sesión general, mediana de 9 (RIC 5-12); la participación en cada una de estas instancias se presenta en la Tabla 1.

Se encontraron 319 autores afiliados a instituciones chilenas, con 637 apariciones, y 23 instituciones con 185 apariciones. Los 10 autores e instituciones con mayor número de presentaciones se exponen en las Tablas 2 y 3 , respectivamente. Cada presentación tuvo un promedio de 4 (2.2) autores afiliados a instituciones chilenas, siendo en un $22.8 \%$ de 4 autores, en $16.5 \%$ de 5 autores y en $13.9 \%$ de 1 y 2 autores, en el $87.3 \%$ de las presentaciones participaron de 1 a 6 autores con afiliación a Chile. El $84.8 \%$ de las presentaciones tuvo afiliación a 1 institución nacional y el $13.9 \%$ a 2 .

En las Tablas 4 y 5 se muestran las áreas temáticas y países en co-autoría con mayor frecuencia de aparición. Un total de 41 presentaciones $(26 \%)$ se realizaron en co-autoría con instituciones extranjeras, siendo en 1 oportunidad con 5 países, en 1 con 2 y en 39 con 1 solo país, las instituciones con mayor cantidad de presentaciones con co-autoría internacional fueron las Universidades de Chile con 11, de Talca con 8 y de Valparaíso con 6.
Tabla 1. Participación chilena en reuniones IADR mundiales, 2001-2012.

\begin{tabular}{|c|c|c|}
\hline Año & Lugar & Participación chilena \\
\hline 2001 & Chiba, Japón. & 0 \\
\hline 2002 & San Diego, California, Estados Unidos. & 5 \\
\hline 2003 & Goteborg. Suecia. & 2 \\
\hline 2004 & Honolulu, Hawaii, Estados Unidos. & 5 \\
\hline 2005 & Baltimore, Estados Unidos. & 10 \\
\hline 2006 & Brisbane, Australia. & 7 \\
\hline 2007 & New Orleans, Estados Unidos. & 11 \\
\hline 2008 & Toronto, Canadá. & 8 \\
\hline 2009 & Miami, Estados Unidos. & 16 \\
\hline 2010 & Barcelona, España. & 25 \\
\hline 2011 & San Diego, California, Estados Unidos. & 12 \\
\hline 2012 & Iguazú, Brasil. & 57 \\
\hline & Total & 158 \\
\hline
\end{tabular}

Tabla 2. Autores de instituciones chilenas con mayor número de presentaciones en reuniones IADR mundiales, 2001-2012.

\begin{tabular}{|c|c|c}
\hline Autores & N & $\%$ \\
\hline Dreyer E & 18 & 11.4 \\
\hline Moncada G & 15 & 9.5 \\
\hline Cifuentes I & 14 & 8.9 \\
\hline Uribe S & 14 & 8.9 \\
\hline Petrasic L & 13 & 8.2 \\
\hline Hernández M & 13 & 8.2 \\
\hline Gamonal J & 9 & 5.7 \\
\hline Martín J & 9 & 5.7 \\
\hline Dutzan N & 8 & 5.1 \\
\hline Fernández E & 8 & 5.1 \\
\hline
\end{tabular}


Tabla 3. Instituciones chilenas con mayor número de presentaciones en reuniones IADR mundiales, 2001-2012.

\begin{tabular}{|c|c|c}
\hline Institución & $\mathbf{N}$ & $\%$ \\
\hline U. de Chile & 68 & 43.0 \\
\hline U. de Talca & 25 & 15.8 \\
\hline Universidad Finis Terrae & 18 & 11.4 \\
\hline U. de los Andes & 17 & 10.8 \\
\hline U. Austral de Chile & 9 & 5.7 \\
\hline U. de Valparaíso & 9 & 5.7 \\
\hline P. U. Católica de Chile & 6 & 3.8 \\
\hline U. de Concepción & 6 & 3.8 \\
\hline U. Andrés Bello & 5 & 3.2 \\
\hline U. Mayor & 4 & 2.5 \\
\hline
\end{tabular}

Tabla 4. Áreas de investigación con mayor número de presentaciones chilenas en reuniones IADR mundiales, 2001-2012.

\begin{tabular}{|c|c|c|}
\hline \multicolumn{1}{|c|}{ Área de Investigación } & N & $\%$ \\
\hline Periodontal & 22 & 13.9 \\
\hline Behavioral, Epidemiologic and Health Services & 20 & 12.7 \\
\hline Cariology & 20 & 12.7 \\
\hline Dental Materials & 16 & 10.1 \\
\hline Prosthodontics & 14 & 8.9 \\
\hline Craniofacial Biology & 11 & 7.0 \\
\hline
\end{tabular}

Tabla 5. Cooperación internacional en las presentaciones chilenas en reuniones IADR mundiales, 2001-2012.

\begin{tabular}{|c|c|c|}
\hline País & N & $\%$ \\
\hline Estados Unidos & 13 & 28.3 \\
\hline Australia & 7 & 15.2 \\
\hline Canadá & 4 & 8.7 \\
\hline Suecia & 4 & 8.7 \\
\hline Brasil & 3 & 6.5 \\
\hline
\end{tabular}

\section{DISCUSIÓN}

En latinoamérica, la práctica científica y la generación de nuevos conocimientos en salud bucal es un bien escaso y costoso, principalmente producto de la falta de una adecuada infraestructura e investigadores con dedicación exclusiva, pero ante todo debido a la ausencia de políticas que estimulen el desarrollo científico del área ${ }^{(12)}$, una situación que afortunadamente ha ido mejorando en los últimos años, liderados por Brasil ${ }^{(7,13)}$.

En el caso chileno, si bien la situación ha mejorado a lo largo de la primera década del presente siglo, aún estamos lejos de los grandes productores a nivel mundial ${ }^{(10)}$. Por otra parte, el explosivo aumento de escuelas de odontología en el país, en su gran mayoría privadas, ha mostrado un impacto casi nulo en la productividad científica de la odontología chilena, medida por el número de publicaciones ISI ${ }^{(11)}$.

Dado lo anterior y considerando la importancia que tienen las reuniones IADR mundiales como instancia de divulgación de las nuevas investigaciones en odontología y generación de redes de cooperación interinstitucional e internacional, es que podemos plantear la participación en dichas reuniones como un indicador valioso para estimar la productividad científica ${ }^{(4,7,14-16)}$, chilena en este caso, y complementar indicadores bibliométricos centrados en las publicaciones en revistas ISI $\left.\right|^{(10,11)}$.

La presente investigación muestra algunos resultados que están en línea con reportes previos en la materia, tales como una concentración y aumento de la producción a lo largo del período estudiado, así como otros que difieren, como la participación de nuevas instituciones odontológicas del país.

Así entonces, encontramos que la Universidad de Chile muestra la mayor participación en reuniones IADR mundiales, tanto a nivel institucional, como de los autores más prolíficos en estas instancias, lo que viene a confirmar los resultados reportados previamente ${ }^{(10,11,14)}$. Una situación similar ocurre en cuanto a las temáticas de las presentaciones, donde Periodoncia ocupa el primer lugar ${ }^{(10,11)}$. También encontramos que la cooperación internacional aún persiste en bajos niveles, solo 1 de cada 4 presentaciones tenía participación de instituciones extranjeras ${ }^{(10)}$.

Las diferencias con investigaciones previas las encontramos en la aparición de 2 universidades privadas, Finis Terrae y de los Andes en el $3^{\text {er }}$ y $4^{\text {to }}$ lugar respectivamente, instituciones que en reportes bibliométricos recientes no tienen mayor participación ${ }^{(10,11)}$. Considerando que las presentaciones en reuniones IADR son una instancia previa a la publicación en revistas de impacto ${ }^{(4,5,15,16)}$, podríamos pensar que en los próximos años estas nuevas instituciones tendrán una mayor participación en la productividad científica ISI de la odontología chilena.

En cuanto a los niveles de participación general, vemos que si bien, existe un aumento a lo largo del período estudiado, este no sigue un patrón regular y podríamos plantear la hipótesis que la participación en reuniones mundiales está más relacionada con facilidades como cercanía geográfica, conectividad aérea, idioma o contacto con investigadores locales, esto considerando que el mayor número de participaciones fueron en Iguazú (Brasil), Barcelona (España) y Miami (Estados Unidos).

Es arriesgado plantear si el nivel de participación chilena en las reuniones mundiales de IADR, medido por el número de presentaciones, es adecuado o no, un factor relevante en esta valoración correspondería a la cantidad de socios de la División Chile, que actualmente es de 175(Consultado en http://iadr.enoah.com/Home/MemberSearch/tabid/9423/Default.aspx el 23 de diciembre de 2012), esto equivale a 0.54 presentaciones por socio durante el último trienio, cifra que aumenta a 0.9 si consideramos todo el período 2001-2012, lo que si bien parece preocupante, no corresponde al panorama completo, ya que no se consideran las presentaciones en la reunión chilena, latinoamericana y otras.

La presente investigación representa una primera aproximación a la valoración de la productividad científica de la odontología chilena en la instancia de mayor importancia internacional, como son las reuniones IADR mundiales ${ }^{(5)}$, y complementa reportes bibliométricos centrados en publicaciones ISI, lo que nos permite tener una visión más completa del área.

Se deben realizar nuevas investigaciones en el área, que permitan cuantificar la participación en otras instancias similares, así como la generación de publicaciones indizadas (ISI, SciELO, Pubmed, Scopus, entre otras) a partir de presentaciones en congresos odontológicos.

\section{CONFLICTOS DE INTERÉS}

No existe relación comercial alguna o financiamiento para la realización de este estudio. 


\section{REFERENCIAS BIBLIOGRÁFICAS}

1. Cartes-Velásquez R. Acceso abierto: Conceptos y aplicación en odontología. Res Dent, 2012; 1 : 2.

2. Manterola C, Pineda V, Vial M, Grande L. ¿Cómo presentar los resultados de una investigación científica? I. La comunicación oral. Cir Esp, 2007; 81(1): 12-17. 3. Manterola C, Pineda V, Vial M, Grande L. ¿Cómo presentar los resultados de una investigación científica? II. El manuscrito y el proceso de publicación. Cir Esp, 2007; 81(2): 70-77.

4. Porter M, McAlpine A, Mkhaliphi D, Grossman E. Subsequent dental research output of South African Division of the IADR Colgate Prize Entrants. J Dent Educ, 2005; 69(11): 1272-1277.

5. Lee DJ, Yuan JC, Prasad S, Barão VA, Shyamsunder N, Sukotjo C. Analysis of abstracts presented at the prosthodontic research section of IADR General Sessions 2004-2005: Demographics, publication rates, and factors contributing to publication. J Prosthodont, 2012; 21(3): 225-231.

6. Collet AM, Jara-Tracchia L, Palacios SB, Itoiz ME. Dental research productivity in Argentina (1993 to 2003). Acta Odontol Latinoam, 2006; 19(2): 81-84.

7. Jara-Tracchia L, Aromando RF, Itoiz ME. Publication: Presentation rate in the Latin American region of the International Association for Dental Research. Acta Odontol Latinoam, 2010; 23(2): 150-152.

8. Collier JM, Vig N, Hammond D. Publish or perish? A survey of abstracts accepted for meetings of the British Association of Oral and Maxillofacial Surgeons, and subsequently published. Br J Oral Maxillofac Surg, 2010; 48(7): 540-543.

9. Abramo G, Ciriaco A, Solazzo M. The relationship between scientists' research performance and the degree of internationalization of their research. Scientometrics, 2011; 86(3): 629-643.
10. Cartes-Velásquez R, Aravena P. Perfil bibliométrico de la odontología chilena 2001-2010. Rev Clin Periodoncia Implantol Rehabil Oral, 2012; 5(1): 5-8.

11. Uribe S, Pradenas I, Urriola M. Impacto del aumento de escuelas de odontología en la productividad científica odontológica chilena. Rev Clin Periodoncia Implantol Rehabil Oral, 2012; 5(1): 13-19.

12. Calatrava L. La producción de conocimientos en salud bucal. Un bien escaso y costoso. Cienc Odont, 2004; 1(2): 117-127.

13. Kaur H, Gupta B. Mapping of dental science research in India: A scientometric analysis of India's research output, 1999-2008. Scientometrics, 2010; 85(1): 361 376.

14. Ruiz B, Urzúa I, Fresno M, Martin J, Moncada G. Productividad científica de grupo de trabajo 2 de la IADR-Chile. Período 2000-2009. Rev Dent Chile, 2010 101(1): 14-19.

15. Lee M, Gardiner J, Federico J, Lane P. A study of productivity and participation at IADR and AADR Annual Meetings from 1970 to 1981. J Dent Res, 1983; 62(5): 578-580.

16. Prasad S, Lee D, Yuan J, Barao V, Shyamsunder N, Sukotjo C. Discrepancies between abstracts presented at International Association for Dental Research Annual Sessions from 2004 to 2005 and full-text publication. Int J Dent, 2012; 2012 859561. 